\title{
BLUE HORIZONTAL BRANCH STARS IN M92 ${ }^{1}$
}

\author{
J. G. COHEN AND J. K. MCCARTHY \\ Palomar Observatory, Mail Stop 105-24, California Institute of Technology, Pasadena, California 91125 \\ Electronic mail: jlc@astro.caltech.edu, jkm@deimos.caltech.edu \\ Received 1996 December 2; revised 1997 January 2
}

\begin{abstract}
We have analyzed high dispersion and high precision spectra of 5 blue horizontal branch stars in the globular cluster M92 to establish that the projected rotational velocity for these stars ranges from 15 to 40 $\mathrm{km} \mathrm{s}^{-1}$. This is larger than that expected based on the rotation of their main sequence progenitors, the spin down of rotation with age, and the conservation of angular momentum. Possible explanations include a rapidly rotating stellar core. An abundance analysis of these spectra of these blue HB stars in M92 yields the same results as have been obtained from the giants in this cluster. There is a hint of a trend of higher abundance as the projected surface rotational velocity increases, which could be chance and requires confirmation. (C) 1997 American Astronomical Society. [S0004-6256(97)01604-X]
\end{abstract}

\section{INTRODUCTION}

There is a large suite of complicated puzzles involving the properties of the horizontal branch in globular clusters. One of these involves the distribution of stars along the horizontal branch, and how that distribution varies from cluster to cluster, i.e., the second parameter problem. (See, for example, Fusi Pecci et al. 1992, 1993.) We believe that the mass loss varies from star to star along the horizontal branch in a given globular cluster, but do not understand what parameters control the mass loss near the tip of the giant branch and at the helium core flash. Another puzzle involves the possible impact of the mixing of the lighter elements, $\mathrm{C}, \mathrm{N}$, and $\mathrm{O}$, and probably $\mathrm{Na}, \mathrm{Mg}$, and $\mathrm{Al}$ as well, produced by nuclear burning in the interior of these evolved stars near the tip of the red giant branch to their surfaces, as reviewed by Kraft (1994). We do not know how, or even if, that is related in some way to the morphology of the horizontal branch. There have also been tantalizing hints of unexpectedly large surface rotational velocities among the horizontal branch stars (Peterson 1983, 1985a, 1985b), but the data available have until recently been very limited.

If conservation of angular momentum prevails, high surface rotation among the red giants in a globular cluster would not be expected due to their large radii, nor is it observed. There is essentially no information on the surface rotation of lower main sequence stars in globulars. High internal rotation would be impossible to detect among lower main sequence stars or among red giants, but might be revealed after mass loss at the helium flash when the stars reach the horizontal branch. It is this phenomenon which we may have detected, although other interpretations are of course possible.

As discussed by Sweigart \& Mengel (1979) and by

${ }^{1}$ Based in large part on observations obtained at the W.M. Keck Observatory, which is jointly operated by the California Institute of Technology and the University of California.
Sweigart (1996), high rotation may influence the process and outcome of mixing, halt diffusion of elements out of the atmosphere, affect the population of the horizontal branch, etc.

The advent of the Keck 10-m Telescope, and its high resolution echelle spectrograph (Vogt et al. 1994), prompted us to re-examine some of these issues.

\section{OBSERVATIONS}

Five blue horizontal branch stars in M92 that were in the outer parts of the cluster (so they would be well isolated spatially) were chosen for study. Charts and $U, B, V$ photometry can be found in Sandage \& Walker (1966) and Sandage (1969). These objects were observed using the HIRES spectrograph on the Keck-1 Telescope in 1994 October. The slit used was 1.1 arcsec wide and 7 arcsec long, which gives a spectral resolution of 34,000 . The detector is a $2048 \times 2048$ pixel Tektronix CCD with a readout noise of 5 electrons rms. The projected slit corresponds to 4 pixels in the dispersion direction. The exposures were binned by a factor of 2 in the direction along the slit prior to readout. Three $1200 \mathrm{sec}$ exposures were obtained for each star; they were analyzed, sky subtracted and summed to form the final spectra. Even at such high dispersion, sky subtraction is particularly vital in the region of the $\mathrm{Na} \mathrm{D}$ lines. The data were reduced using Figaro.

The spectra cover the wavelength range from 4304 to $6630 \AA$ (orders 82 to 54). With the current HIRES CCD detector, there is full spectral coverage at the blue end $(\lambda<5000 \AA$ ), but gaps of about $30 \%$ of the apparent order length develop at the red end.

The radial velocity stability of HIRES is excellent as it is permanently mounted in a temperature stabilized housing on one of the Nasmyth platforms of the Keck-1 Telescope. A Th-Ar lamp was used for wavelength calibration.

The precision of these data is very high. The signal-tonoise ratio, measured by looking at the statistics in con- 


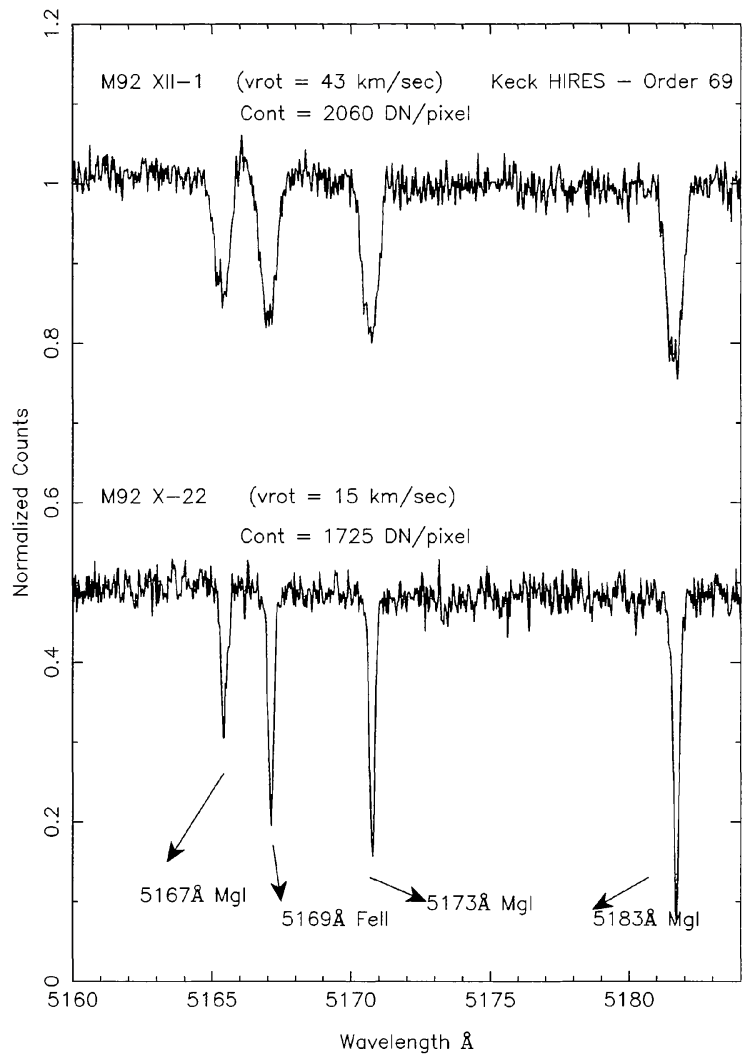

FIG. 1. A $24 \AA$ section of spectrum from order 69 from the HIRES spectrum of the blue HB stars XII-1 and X-22 in the globular cluster M92. The spectra have been normalized to the continuum values indicated in each panel. The spectrum of M92 X-22 has been vertically shifted by 0.5 . The projected rotational velocities are indicated.

tinuum segments $5 \AA$ long near the center of each order that appear to be line free, is between 40 and 70 per pixel ( 80 to 140 per resolution element). This is an underestimate because of the probable presence of undetected weak absorption features within each segment.

\section{ROTATIONAL VELOCITIES}

In all earlier work, mostly by Ruth Peterson and her collaborators (Peterson 1983, 1985a, 1985b) where the spectral resolution and the signal-to-noise ratio were lower, the rotational broadening had to be inferred from the width of the peak of the cross correlation between the spectrum of a rotating star and that of one believed to have little or no rotation. Peterson et al. (1995) succeeded in resolving the lines of the infrared $\mathrm{O}$ I triplet in blue $\mathrm{HB}$ stars in several globular clusters, but because the $\mathrm{O}$ I triplet lines are on the saturated part of the curve of growth, the determination of rotation is coupled to the abundance of oxygen, which varies widely from star to star in globular clusters as described by, for example, Sneden et al. 1991. Hence the solution for $v_{\text {rot }}$ becomes quite complex. However, in our case the profiles of much weaker absorption lines could be detected, and it was apparent from an initial inspection of the raw data at the telescope as well as of the reduced HIRES spectra that the rotational velocities were large and differed significantly

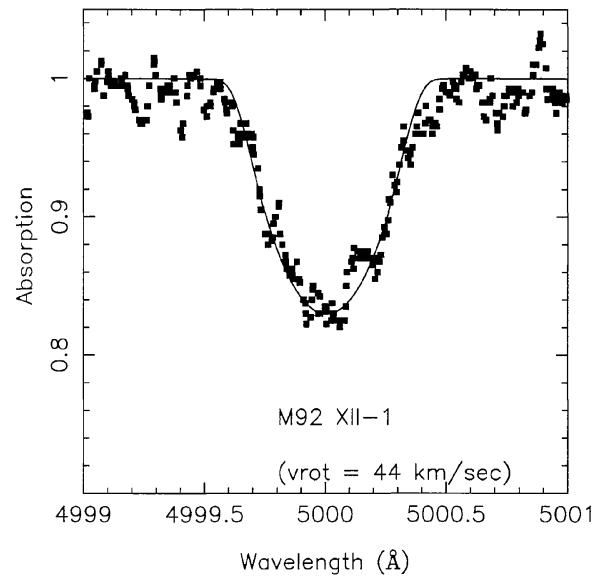

FIG. 2. The points represent the scaled, shifted and overlaid profiles of four absorption lines in the blue HB star M92 XII-1, with the line center set to $5000 \AA$ in each case. A four point running mean is shown. The solid line is the profile of an $\mathrm{Th}-\mathrm{Ar}$ arc emission line broadened by a projected rotational velocity of $44 \mathrm{~km} \mathrm{~s}^{-1}$.

from star to star. Figure 1 illustrates the comparison over a $24 \AA$ region of the spectrum of the most rapidly rotating M92 horizontal branch star in our sample (M92 XII-1) to that of one of the slowly rotating stars. The equivalent width of the weakest line marked in each panel, the $5167 \AA \mathrm{Mg}$ I line, is $39 \mathrm{~m} \AA$ for M92 X-22 and $86 \mathrm{~m} \AA$ for M92 XII-1. The spectra are normalized to the continuum, whose value in DN $\left(1 \mathrm{DN} \approx 2 e^{-}\right)$is given in the panels of the figure. The mean FWHM of isolated lines in the Th-Ar arc is $8.6 \pm 0.3 \mathrm{~km}$ $\mathrm{s}^{-1}$.

In our spectra, in part because of the very low metallicity of M92, the absorption lines are weak and in most cases well isolated. The stellar absorption lines which were used to determine the rotation ranged in equivalent width from 20 to $120 \mathrm{~m} \AA$. This means that these lines are largely on the linear part of the curve of growth, and that their intrinsic profiles are identical, scaled of course to varying total absorption. Damping parameters and abundances of the line absorber are irrelevant to the shape of the normalized line profile.

Two methods of determining the rotational velocity were used. The first relied on measuring the width of the best fitting Gaussian profiles for a number of isolated, unblended absorption features. Between 5 and 15 lines were used for each star. These were compared to similar measurements of the profiles of the arc emission lines, broadened by varying amounts of rotational broadening. The rotational broadening profile adopted is that of Unsöld (1955). A value for the limb darkening coefficient of $\beta=2.7$ was used, based on Allen (1973). The results are insensitive to a change in $\beta$ by a factor of 2. This method did not work well for the most rapidly rotating stars, where a Gaussian does not provide an adequate fit to the observed line profiles.

The second method was to choose a set of four reasonably strong stellar absorption lines in each star, shift and scale their profiles appropriately to form a composite line profile for each M92 HB star, $Y_{\text {star }}$. The appropriate scaling was done in the wavelength domain as well. The result of this process was then compared with the profile of a single Th-Ar 
TABLE 1. Projected rotational velocities for blue horizontal branch stars in M92.

\begin{tabular}{lccccc}
\hline \hline & $\begin{array}{c}v_{\text {rot }}(\sin (i)) \\
\text { Gaussian } \\
\left(\mathrm{km} \mathrm{s}^{-1}\right)\end{array}$ & $\begin{array}{c}\sigma \\
\left(\mathrm{km} \mathrm{s}^{-1}\right)\end{array}$ & $\begin{array}{c}v_{\text {rot }}(\sin (i)) \\
\text { Profile Fit } \\
\left(\mathrm{km} \mathrm{s}^{-1}\right)\end{array}$ & $\begin{array}{c}\sigma \\
\left(\mathrm{km} \mathrm{s}^{-1}\right)\end{array}$ & $\begin{array}{c}V_{r} \\
\left(\mathrm{~km} \mathrm{~s}^{-1}\right)\end{array}$ \\
\hline IV-17 & 14 & 2 & 15 & 4 & -127.6 \\
IV-27 & 22 & 3 & 27 & 4 & -115.7 \\
X-22 & 13 & 2 & 15 & 3 & -121.6 \\
XII-1 & 37 & 4 & 43 & 5 & -127.6 \\
XII-9 & 22 & 3 & 29 & 3 & -127.7 \\
\hline \hline
\end{tabular}

arc emission line, broadened by varying amounts of rotation, $Y(\operatorname{arc})_{\text {broad }}$. A statistical measure of the agreement of the two profiles was computed, although the final rotational velocity assigned using this method was chosen by eye after examining plots with $v_{\text {rot }}(\sin (i))$ near that giving the minimum error. An example of this for the M92 blue HB star in our sample with the largest projected $v_{\text {rot }}$ is shown in Fig. 2 . The lines included, with their equivalent widths in M92 XII-1, are 4383.6 (Fe I, $86 \mathrm{~m} \AA$ ), 4404.8 (Fe I, $55 \mathrm{~m} \AA$ ), 4468.5 (Ti II, $108 \mathrm{~m} \AA$ ), and $4572.0 \AA$ (Ti II, $77 \mathrm{~m} \AA$ ). A four point running mean of the shifted, scaled, and overlaid profiles is displayed.

The results are listed in Table 1 . The first column gives the star name (from Sandage \& Walker 1966), the second gives the rotational velocity inferred from the Gaussian fits, the third column gives the rotational velocity inferred from the composite line profile fitting followed by its error. The errors in the first case are the $1 \sigma \mathrm{rms}$ errors for the group of lines, while in the second case the errors are based on $\Sigma\left[Y(\operatorname{arc})_{\text {broad }}-Y_{\text {star }}\right]^{2}$ evaluated at $0.01 \AA$ intervals over the region with absorption greater than $3 \%$ of the continuum, calculated for each value of $v_{\text {rot }}$. The agreement between the two independent determinations of $v_{\text {rot }}(\sin (i))$ is gratifying.

There is no correlation between projected rotational velocity and $T_{\text {eff }}$.

We would like to know if the distribution of $v_{\text {rot }}(\sin (i))$ is consistent with a constant value of $v_{\text {rot }}$ and a random distribution of the angle of inclination of the axis of rotation with respect to the line of sight. A $\chi^{2}$ test applied to these data indicates a probabilty of $\approx 10 \%$ that the sample of blue HB stars in M92 is derived from such a population with a uniform rotation of about $45 \mathrm{~km} \mathrm{~s}^{-1}$. A larger sample could refine (or refute) this statement.

The final column of Table 1 gives the heliocentric radial velocities. As expected, all the stars are members of M92 with $\left\langle v_{r}\right\rangle=-123.7 \mathrm{~km} \mathrm{~s}^{-1}$ and $\sigma=5.1 \mathrm{~km} \mathrm{~s}^{-1}$. This velocity dispersion is in excellent agreement with that determined by Lupton et al. (1985) from a much larger sample of stars in M92.

\section{DISCUSSION OF THE ROTATIONAL VELOCITIES}

The main sequence progenitors of the blue horizontal branch stars in M92 have a mass of about $0.85 \mathrm{M}_{\odot}$ and are late $\mathrm{F}$ to early $\mathrm{G}$ dwarfs. The rotation of main sequence stars has been a subject of interest since the work of Kraft (1967). For many nearby stars, periods are now available which yield the rotation velocity itself rather than the projected ve- locity of rotation. These have come from extensive monitoring of the variability of the chromospheric emission in the core of the $H$ and $K$ lines (Baliunas \& Vaughan 1985). Benz et al. (1984) showed that in clusters as young as the Hyades, $\left\langle v_{\text {rot }}\right\rangle$ is less than $12 \mathrm{~km} \mathrm{~s}^{-1}$ for 10 stars with spectral types ranging from $\mathrm{F} 8 \mathrm{~V}$ to $\mathrm{G} 5 \mathrm{~V}$. $v_{\text {rot }}$ as large as $50 \mathrm{~km} \mathrm{~s}^{-1}$ is not reached in the Hyades until the spectral type is F5 V, too early to be the progenitors of the blue HB stars in M92. We assume that metallicity differences do not significantly perturb this picture.

In addition, it is clear from studying open clusters of varying ages younger than the Hyades that there is a spin-down of rotation with time for these stars, where the rotational decay due to magnetic braking is predicted to be of the form $t^{-0.5}$ (Skumanich 1972; Endal \& Sofia 1981). Whether and how far this extends beyond the age of the Hyades is not known, but extrapolating blindly from $7 \times 10^{8}$ years (the determination of the age of the Hyades by Mazzei \& Pigatto 1988) to ages characteristic of globular clusters, one expects a further spin-down of the rotational velocity of a factor of $\approx 4$.

Based on models of horizontal branch evolution, the mean mass of the M92 blue HB stars is about $0.70 \mathrm{M}_{\odot}$, with a scatter of perhaps $0.03 \mathrm{M}_{\odot}$. (Crocker \& Rood 1988; Lee et al. 1990). We assume solid body rotation and ignore any further spin-down for the main sequence stars beyond that shown in the Hyades. We assume that the angular momentum/gm in the $\approx 0.15 \mathrm{M}_{\odot}$ lost during stellar evolution between the main sequence and the $\mathrm{HB}$ is the same as that of the initial star. The radii of these blue $\mathrm{HB}$ stars are $\approx 3$ $\mathrm{R}_{\odot}$, and we find that the expected rotational velocity for blue HB stars should be under $10 \mathrm{~km} \mathrm{~s}^{-1}$.

Our projected rotational velocities, as well as the latest work by Peterson et al. (1995), show that velocities exceeding those derived under the assumptions above are common among the blue HB stars in globular clusters. Among the many possibilities that could explain these results is that main sequence stars have a more rapidly rotating core, and as mass is lost, a higher surface rotation is seen. The evolution of differentially rotating stars is discussed by Pinsonnealt et al. (1991). Peterson et al. (1995) gives a detailed discussion of the myriads of other possible explanations.

Several recent studies at high dispersion of field RR Lyrae stars (Clementini et al. 1995; Lambert et al. 1996; Peterson et al. 1996; Fernley \& Barnes 1996) demonstrate that high rotation is not seen in any of these objects, and the sample of field RR Lyrae stars observed at high dispersion is now reasonably large. The only time that line broadening can be detected is near $\phi \approx 0.85$, and this presumably arises from the effect of shocks. These variables are HB stars only slightly cooler than the coolest of the blue HB stars in M92 that we examine here. We speculate this is related to the fact that in one case we are looking at field stars with a relatively young mean age, and in the other we are looking at much older objects, although it is also possible that globular cluster stars have more angular momentum ab initio than do field halo stars. This difference is quite puzzling. 
TABLE 2. Atmospheric parameters for M92 blue HB stars.

\begin{tabular}{ccccccccc}
\hline \hline Star & $\begin{array}{c}K \\
(\mathrm{mag})\end{array}$ & $\begin{array}{c}T_{\text {eff }}(B-V) \\
(\mathrm{K})\end{array}$ & $\begin{array}{c}\delta T_{\text {eff }}(B-V)^{\mathrm{a}} \\
(\mathrm{K})\end{array}$ & $\begin{array}{c}T_{\text {eff }}(V-K) \\
(\mathrm{K})\end{array}$ & $\begin{array}{c}\delta T_{\text {eff }}(V-K)^{\mathrm{b}} \\
(\mathrm{K})\end{array}$ & $\begin{array}{c}\text { Adopted } T_{\text {eff }} \\
(\mathrm{K})\end{array}$ & $\begin{array}{c}T_{\text {eff }}\left(H_{\gamma}\right) \\
(\mathrm{K})\end{array}$ & $\begin{array}{c}\log (g) \\
(\mathrm{dex})\end{array}$ \\
\hline $\mathrm{IV}-17$ & 15.44 & 8750 & $(+750,-200)$ & 10000 & $(+1000,-750)$ & 9375 & $>8250$ & 3.6 \\
$\mathrm{IV}-27$ & 14.57 & 7500 & $(+300,-200)$ & 7625 & $(+250,-250)$ & 7550 & $7500 \pm 100$ & 3.1 \\
X-22 & 14.46 & 7500 & $(+300,-200)$ & 7450 & $(+250,-250)$ & 7450 & $7500 \pm 100$ & 3.1 \\
XII-1 & 14.31 & 7400 & $(+200,-200)$ & 7250 & $(+250,-250)$ & 7325 & $7250 \pm 100$ & 3.0 \\
XII-9 & 14.36 & 7600 & $(+200,-200)$ & 7375 & $(+250,-250)$ & 7500 & $7450 \pm 100$ & 3.1 \\
\hline \hline
\end{tabular}

${ }^{\mathrm{a}}$ Uncertainty in $T_{\text {eff }}$ from $\delta(B-V)= \pm 0.04 \mathrm{mag}$.

${ }^{b}$ Uncertainty in $T_{\text {eff }}$ from $\delta(V-K)= \pm 0.10 \mathrm{mag}$.

\section{ATMOSPHERIC PARAMETERS}

In preparation for an analysis of the spectral features we need to determine the atmospheric parameters for these stars. Photoelectric $U, B, V$ photometry already exists (Sandage $1969)$, and we supplement that with photometry at $K(2.2$ $\mu \mathrm{m}$ ) using the new infrared camera (Murphy et al. 1995) at the 60-inch telescope on Palomar Mountain. These measurements were kindly made and reduced by Mike Pahre of Caltech. An aperture 12 arcsec in diameter was used. The typical photometric uncertainty is $0.05 \mathrm{mag}$. Assuming the metallicity is $[\mathrm{Fe} / \mathrm{H}]=-2.35 \mathrm{dex}$ (Cohen 1979), the observed $B-V$ and $V-K$ colors, corrected for $\mathrm{E}(B-V)=0.02$ mag (Sandage 1969), were used together with the colors predicted from Kurucz's (1993) grid of LTE model atmospheres.

This procedure yields the temperatures given in Table 2 . The $T_{\text {eff }}$ values from $V-K$ are given double weight in determining the $T_{\text {eff }}$ used in the analysis because they are much less sensitive to small observational errors in the photometry. The observed $K$ magnitudes are listed in the second column.

The surface gravities in Table 2 were calculated once $T_{\text {eff }}$ was determined, given that the mass and luminosity of the stars were known. (The distance modulus adopted was $7.85 \mathrm{kpc}$ from Cohen 1992 and $\mathrm{E}(B-V)=0.02$ mag was used.)

In addition, the profile of $H_{\gamma}$ was available to confirm that the choice of $T_{\text {eff }}$ and surface gravity were reasonable. While $H_{\beta}$ is within the wavelength range, the constraints of defining its continuum in this echelle format made it less reliable to use, and $H_{\gamma}$, formed deeper in the atmosphere, should be the better diagnostic. The procedure for defining the continuum for the broad Balmer lines in this echelle format is described in detail in McCarthy \& Nemec (1997).

In the temperature regime around $T_{\text {eff }} \sim 7500 \mathrm{~K}$, the $H_{\gamma}$ profile is much more sensitive to small variations in $T_{\text {eff }}$ than to small variations in $\log (g)$. We define $W\left(H_{\gamma}\right)$ as the equivalent width of the Balmer line measured over the regime from $1 \AA$ from the line center (to avoid the core itself) to $16 \AA$ from the line center, beyond which the profile is very difficult to determine due to the echelle format of the observations. In this regime, for an increase in $T_{\text {eff }}$ of $250 \mathrm{~K}$, $W\left(H_{\gamma}\right)$ calculated from the Kurucz grid of theoretical Balmer line profiles increases by $12 \%$, while it only increases by $3 \%$ for $\Delta(\log (g))=0.5$ dex.

Since the luminosity and mass of these blue HB stars in M92 are rather tightly constrained, a change in $T_{\text {eff }}$ of $20 \%$, which would easily be detectable, is required to change the surface gravity by a factor of two. We will see that in deduced parameters such as the abundances, the temperature uncertainties will dominate. Table 2 lists in the penultimate column $T_{\text {eff }}$ determined from $H_{\gamma}$ for the four cooler blue HB stars. The very good agreement further validates the choice of atmospheric parameters given in Table 2. (The hottest star has much broader Balmer line profiles and the continuum determination thus becomes more difficult.)

The distinction in color and in Balmer line profiles for a fixed $T_{\text {eff }}$ and $\log (g)$ between the $[\mathrm{Fe} / \mathrm{H}]=-2.0 \operatorname{dex}$ and -2.5 dex grid of models is not significant.

\section{ABUNDANCES ON THE HB OF M92}

\subsection{Motivation and History}

Abundance variations in $\mathrm{C}, \mathrm{N}$, and $\mathrm{O}$ have been known to occur within globular clusters for many years and have always been ascribed to mixing. Carbon et al. (1982), Pilachowski (1988), and Sneden et al. (1991) have studied various aspects of this problem for large samples of giants in M92. It is now clear that $\mathrm{Na}, \mathrm{Mg}$, and $\mathrm{Al}$ variations also occur among red giants within individual globular clusters (see, for example, Pilachowski et al. 1996, who discuss $\mathrm{Na}$ and $\mathrm{Mg}$ abundances in 130 giants in M13) and that they too are the result of mixing of nuclear-processed material from the stellar interior. Until Denisenkov \& Denisenkova's seminal paper (1990), no one understood how to produce $\mathrm{Na}$ and $\mathrm{Al}$ in such low mass and relatively unevolved stars. (See also Langer et al. 1993.) The interaction between internal rotation and mixing is a critical one (Sweigart \& Mengel 1979, Sweigart 1996), as is that between rotation and diffusion in the photosphere.

Detailed abundance analyses of M92 giants for the heavier elements, which should be unmodified from the original formation of the globular cluster for stars not evolved significantly beyond the He flash, have been performed by many groups, beginning with Helfer et al. (1959), then Cohen (1979), Peterson et al. (1990), Sneden et al. (1991) for a few selected elements, and Armosky et al. (1994) for the heavy $s$-process elements.

We analyze the abundances of several elements in the blue HB stars in M92 for which we have HIRES spectra. We do this not to contribute to the abundance determination for M92, which we regard as well established by the many previous investigations utilizing high dispersion spectra and modern model atmosphere analysis techniques applied to the 
TABLE 3. Heavy element abundances for four blue horizontal branch stars in M92.

\begin{tabular}{lccc}
\hline \hline Element & $N_{\text {lines }}$ & $\begin{array}{c}{[\mathrm{X}]^{\mathrm{a}}} \\
(\mathrm{dex})\end{array}$ & $\begin{array}{c}\sigma^{\mathrm{b}} \\
(\mathrm{dex})\end{array}$ \\
\hline $\mathrm{Na} \mathrm{I}$ & 2 & -2.42 & 0.36 \\
$\mathrm{Mg}$ I & 4 & -1.92 & 0.22 \\
$\mathrm{Mg}$ II & $1^{\mathrm{c}}$ & -1.95 & 0.07 \\
$\mathrm{Ca}$ I & 1 & -2.02 & 0.25 \\
$\mathrm{Ti}$ II & 9 & -1.90 & 0.18 \\
$\mathrm{Cr}$ II & 2 & -1.96 & 0.16 \\
$\mathrm{Fe}$ I & 5 & -2.34 & 0.19 \\
$\mathrm{Fe}$ II & 7 & -2.26 & 0.24 \\
$\mathrm{Ba} \mathrm{II}$ & 1 & -2.50 & 0.25 \\
\hline \hline
\end{tabular}

${ }^{a}[\mathrm{X}]=\log 10(X)($ M92 star $)-\log 10(X)($ Sun $)$.

${ }^{b}$ This is the standard deviation of the abundances for the four stars about the mean, not the standard error of the mean itself.

cThis is the close doublet of $\mathrm{Mg}$ II at $4481 \AA$.

red giants. Instead we intend to demonstrate, in preparation for moving to much more metal-rich globular clusters, that reliable abundances can be obtained from these HB stars, and that our normalizations and procedures are correct. Furthermore, we need to establish that in contrast to the situation with the hotter blue HB stars such as was found by Glaspey et al. (1989), there is no evidence for gravitational settling among these cooler blue HB stars. Lambert et al. (1992) have also explored these issues, recent models for meridional circulation and diffusion are given by Charbonneau \& Michaud (1991) among others, and Vauclair \& Vauclair (1982) review the taxonomy of hot stars with peculiar abundances that are believed to have arisen as a result of element segregation in stellar photospheres.

\subsection{Our Abundances for the Blue HB Stars in M92}

We have adopted the table of transition probabilities of Luck (1992) updated to include the modifications for Fe I suggested by Lambert et al. (1996) and those for Fe II from Biémont et al. (1991). The atmospheric parameters adopted are those of Table 2. The Kurucz (1993) grid of model atmospheres was used together with the MOOG code of Sneden (1975). Using the solar model supplied by Kurucz (1993) and the solar equivalent widths of Moore et al. (1966), we can reproduce the solar abundances satisfactorily.

We do not use any non-LTE corrections. Clementini et al. (1995), Lambert et al. (1996) and Fernley \& Barnes (1996) discuss this issue for field RR Lyrae stars whose atmospheric parameters are fairly close to those of our M92 blue HB stars. The corrections for non-LTE ionization equilibrium are fairly small $(<0.2)$ dex, but may become larger with decreasing metallicity as the photoionizing UV flux increases. In any case, our final results (Table 3 ) give no evidence that a substantial correction is necessary. No corrections for hyperfine structure were applied to any lines either. A microturbulence velocity of $2.0 \mathrm{~km} \mathrm{~s}^{-1}$ was adopted.

One of the 5 blue HB stars in M92 is significantly hotter than the other four stars, and its lines are much weaker. Since M92 is a very metal poor globular cluster, this makes all the
TABLE 4. Possible correlation of abundance and rotation.

\begin{tabular}{lcc}
\hline \hline Star(s) & $v_{\text {rot }}(\sin (i))$ Group & {$[\langle\mathrm{A}\rangle]$} \\
\hline $\mathrm{X}-22$ & Low & -0.26 \\
$\mathrm{IV}-27+\mathrm{XII}-9$ & Intermediate & $0^{\mathrm{a}}$ \\
XII-1 & High & +0.13 \\
\hline \hline
\end{tabular}

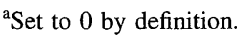

lines in that star extremely weak. We therefore only consider the four cooler stars. Table 3 gives the number of lines used, the mean abundance for each element and the $\sigma$ about the mean for the abundance of each element derived from these 4 stars. Except for Na I, $\sigma \leqslant 0.25$ dex for each ion included, which is comparable to the observational and modelling errors. Because only the $\mathrm{Na} \mathrm{D}$ doublet lines were used, the $\mathrm{Na}$ abundance is highly sensitive to errors in $T_{\text {eff }}$, and the large $\sigma$ may be a reflection of this as well as of a potential range in abundance of $\mathrm{Na} / \mathrm{Fe}$.

The abundances of Table 3 are in good agreement with those of Cohen (1979) and of the most recent published analyses by Peterson et al. (1990), Sneden et al. (1991), and Armosky et al. (1994). Sneden et al. (1991) find $[\mathrm{Fe} / \mathrm{H}]$ $=-2.25 \pm 0.02$ dex from 9 red giants in M92, while we obtain -2.29 dex. (The fifth and hottest M92 blue HB star gives -2.35 dex, from $\mathrm{Fe}$ II lines only.) We therefore are confident that our procedure is sound and that when we proceed to analyze HB stars with similar $T_{\text {eff }}$ in much more metal-rich globular clusters the results should be valid.

\subsection{Abundance and Rotation}

There is evidence for a small correlation between abundance and projected rotational velocity. (If this is true, then the projected rotational velocity must be a good indicator of the true rotational velocity and not just indicate inclination angle.) As our best abundance indicator, we take the average of the best determined elements, namely $[[\mathrm{Mg}](\mathrm{Mg} \mathrm{I})$ $+[\mathrm{Mg}](\mathrm{Mg} \mathrm{II})],[\mathrm{Ti}](\mathrm{Ti} \mathrm{II})$, and $[[\mathrm{Fe}](\mathrm{Fe} \mathrm{I})+[\mathrm{Fe}](\mathrm{Fe} \mathrm{II})]$. The average of those three quantities we denote $[\langle\mathrm{A}\rangle]$.This is given in Table 4 for the star with the highest $v_{\text {rot }}(\sin (i))$, the two stars with intermediate rotation, and the star with the smallest projected rotation. The hottest M92 blue HB star is ignored.

The equivalent widths are of high accuracy here; the accuracy of the $T_{\text {eff }}$ determinations is the limiting factor in our ability to isolate small but real abundance differences from star to star. The possible trend of increasing $[\mathrm{A} / \mathrm{H}]$ with increasing rotation urgently requires confirmation with a larger sample of HB stars in M92.

\section{SUMMARY}

We have analyzed high dispersion and high precision Keck/HIRES spectra of 5 blue horizontal branch stars in the globular cluster M92 to establish that the projected rotational velocity for these stars ranges from 15 to $40 \mathrm{~km} \mathrm{~s}^{-1}$. This is larger than that expected based on the assumed rotation of their main sequence progenitors, the spin down of rotation 
with age, and the conservation of angular momentum. Possible explanations include a rapidly rotating stellar core.

An abundance analysis of these spectra of blue HB stars in M92 yields the same results obtained from the giants in this cluster. There is evidence of a small increase in abundance of the heavier elements as $v_{\text {rot }}(\sin (i))$ increases, but the sample urgently needs to be enlarged.

We now feel confident that our methods are valid and that we are ready to proceed to analyze the spectra of HB stars in much more metal-rich globular clusters.
We are grateful to R. Earle Luck for providing the computer-ready compendium of $g f$-values as well as for the LTE model atmosphere and abundance analysis codes provided by Bob Kurucz and Chris Sneden. We are grateful to Mike Pahre for providing the infrared photometry for the M92 blue HB stars. It is a pleasure to thank the W.M.Keck Foundation, and its late President Emeritus, Howard B. Keck, for the generous grant that made the Keck Observatory possible. We thank Theresa Chelminiak for observing assistance at the telescope.

\section{REFERENCES}

Allen, C.W. 1973, Astrophysical Quantitites, 3rd ed. (Athlone Press, London)

Armosky, B. J., Sneden, C., Langer, G. E., \& Kraft, R. P. 1994, AJ, 108, 1364

Baliunas, S. L., \& Vaughan, A. H. 1985, ARA\&A, 23, 379

Benz, W., Mayor, M., \& Mermilliod, J. C. 1984, A\&A, 138, 93

Biémont, E., Baudoux, M., Kurucz, R. L., Ansbacker, W., \& Pennington, E. H. 1991, A\&A, 249, 359

Carbon, D. F., Langer, G. E., Butler, D., Kraft, R. P., Suntzeff, N. B.,

Kemper, E., \& Trefzger, C. F. 1982, ApJS, 49, 207

Charbonneau, P., \& Michaud, G. 1991, ApJ, 370, 693

Clementini, G., Carretta, E., Gratton, R., Merighi, R., Mould, J. R., \& McCarthy, J. K. 1995, AJ, 110, 2319

Cohen, J. G. 1979, ApJ, 231, 751

Cohen, J. G. 1992, ApJ, 400, 528

Crocker, D. A., \& Rood, R. T. 1988, ApJ, 332, 236

Denisenkov, P. A., \& Denisenkova, S. N. 1990, SvAL, 16, 275

Endal, A. S., \& Sofia, S. 1981, ApJ, 243, 625

Fernley, J., \& Barnes, T. G. 1996, A\&A, 312, 957

Fusi Pecci, F., Corsi, C. E., Cacciari, C., \& Buonanno, R. 1992, AJ, 104 1831

Fusi Pecci, F., Ferraro, F. R., Bellazzini, M., Djorgovski, S., Piotto, G., \& Buonanno, R. 1993, AJ, 105, 1145

Glaspey, J. W., Michaud, G., Moffat, A. F. J., \& Demers, S. 1989, ApJ, 339, 926

Helfer, H. L., Wallerstein, G., \& Greenstein, J. L. 1959, ApJ, 129, 700

Kraft, R. P. 1967, ApJ, 150, 551

Kraft, R. P. 1994, PASP, 106, 553

Kurucz, R.L. 1993, CD-Rom No. 13, Smithsonian Astrophysical Observatory

Lambert, D. L., Heath, J. E., Lemke, M., \& Drake, J. 1996, ApJS, 103, 183

Lambert, D. L., McWilliam, A., \& Smith, V. V. 1992, ApJ, 386, 685
Langer, G. E., Hoffman, R., \& Sneden, C. 1993, PASP, 105, 301

Lee, Y. W., Demarque, P., \& Zinn, R. 1990, ApJ, 350, 155

Luck, R.E. 1992 (private communication)

Lupton, R., Gunn, J. E., \& Griffin, R. F. 1985, in Dynamics of Globular Clusters, IAU Symposium No.113, edited by J. Goodman \& P. Hut (Reidel, Dordrecht), p. 19

Mazzei, P., \& Pigatto, L. 1988, A\&A, 193, 148

McCarthy, J. K., \& Nemec, J. M. 1997, ApJ, in press

Moore, C.E., Minnaert, M.G.J., \& Houtgast, J. 1966, NBS Monograph 61

Murphy, D. C., Persson, S. E., Pahre, M. A., Sivaramakrishnan, A., \& Djorgovski, S. G. 1995, PASP, 107, 718

Peterson, R. C. 1983, ApJ, 275, 737

Peterson, R. C. 1985a, ApJ, 289, 320

Peterson, R. C. 1985b, ApJ, 294, L35

Peterson, R. C. Kuruzc, R. L., \& Carney, B. W. 1990, ApJ, 350, 173

Peterson, R. C. Rood, R. T., \& Crocker, D. A. 1995, ApJ, 453, 214

Peterson, R. C., Carney B. W., \& Latham, D. W. 1996, ApJ, 465, L47

Pilachowski, C. A. 1988, ApJ, 325, L57

Pilachowski, C. A., Sneden, C., Kraft, R. P., \& Langer, G. E. 1996, AJ, 112, 545

Pinsonneault, M. H., Deliyannis, C. P., \& Demarque, P. 1991, ApJ, 367, 239

Sandage, A. R. 1969, ApJ, 157, 515

Sandage, A. R., \& Walker, M. F. 1966, ApJ, 143, 313

Skumanich, A. 1972, ApJ, 171, 565

Sneden, C., 1975, Ph.D. thesis, University of Texas

Sneden, C., Kraft, R. P., Prosser, C. F., \& Langer, G. E. 1991, AJ, 102, 2001

Sweigart, A. V., \& Mengel, J. G. 1979, ApJ, 229, 624

Sweigart, A.V. 1996, preprint

Unsöld, A. 1955, Physik der Sternatmospharen 2nd ed. (Springer, Berlin)

Vauclair, S., \& Vauclair, G. 1982, ARA\&A, 20, 37

Vogt, S., et al. 1994, SPIE, 2198, 362 